\title{
Carboxylated Cellulose Nanocrystals as Environmental-Friendly and Multi-Functional Additives for Bentonite Water-Based Drilling Fluids Under High-temperature Conditions
}

Xinliang Li ( $\nabla$ petroleumliang@outlook.com)

Southwest Petroleum University

Kai Wang

China National Petroleum Corp Chuanqing Drilling Engineering Co Ltd

\section{Research Article}

Keywords: cellulose nanocrystals, water-based drilling fluid, shale gas drilling, rheology, fluid loss, inhibition

Posted Date: October 28th, 2021

DOl: https://doi.org/10.21203/rs.3.rs-1009566/v1

License: () (1) This work is licensed under a Creative Commons Attribution 4.0 International License. Read Full License 


\section{Abstract}

During the oil and gas drilling engineering, the selection of drilling fluids must take account of the technical and environmental factors. This study investigated the effectiveness of carboxylated cellulose nanocrystals (denoted as CNCs) as environmentally friendly additives in improving the rheological, filtration, and inhibitive performances of bentonite (BT) water-based drilling fluids (WBDFs). CNCs used in this study were modified by carboxylation reaction, displaying small size, negative surface charge, good colloidal stability, and prominent shear thinning behavior. The experimental results indicated that BT/CNC suspensions had superior rheological properties, low fluid loss volumes, and effective inhibition, even at $140{ }^{\circ} \mathrm{C}$. Microstructure analysis demonstrated that CNCs could attach to the surface of BT via hydrogen bond and ionic bond. CNCs, BT, and vicinal water molecules could form a stiff gel network, which had a strong resistance to flow under shear force, leading to a significant improvement in the rheological properties. Moreover, under the differential pressure, BT/CNC suspensions formed thin and less hydrophilic filter cakes with compact layered structure, thereby efficiently decreasing the fluid loss volume. Finally, due to the gel network and filtration ability, BT/CNC suspensions performed low water activity, which was beneficial for preventing the penetration of free water into the shales and borehole well. Thus CNCs also exerted satisfactory inhibition on hydration and dispersion of BT and shales. As a result, CNCs showed great potential to be used as efficient, multi-functional, and environmentally friendly additives in WBDFs.

\section{Introduction}

Drilling fluids are vital functional fluids in oil and gas drilling operations(R Caenn and Chillingar 1996). They can cool and lubricate drilling tools, carry cuttings from the bottom of borehole to the surface, stabilize borehole, and thereby ensure the success of the drilling operations(Adeleye Sanmi Apaleke 2012, Agwu et al. 2021). With the increasing strict technical and environmental requirements in complex formations, the selection of drilling fluids must take account of the thermal stability, salt tolerance, cost, health safety, and environmental factor, especially in shale areas(Amanullah et al. 2016). In these easy-hydrated areas, oil-based drilling fluids (OBDFs) have been often applied due to their excellent technical performance, such as good inhibition, lubricity, filtration, and high-temperature resistant(Patel et al. 2007). However, OBDFs are expensive, disadvantageous to well logging, and easy to pollute environment(SJ Chegny 2008). Therefore, high-performance WBDFs with environmental consideration are being emphatically studied to replace the OBDFs(M Attia, W Elsorafy and D'Angelo 2010).

To achieve ideal properties, WBDFs are usually composed of water, clay, fluid loss additive, rheology modifier, inhibitor, and other organic and inorganic additives(Yao et al. 2014, Akpan et al. 2019). Effective fluid loss additives include copolymers of acrylamide (AM), 2-Acrylamido -2-methylpropane sulfonic acid (AMPS) and cationic monomers(Liu et al. 2016, Yang et al. 2017), sulfonated phenolic resin(Sun, Xin and Hu 2013, Liu et al. 2018), modified asphalt(Igwilo et al. 2020), and others. Xanthan gum(Villada et al. 2017) and polyanionic cellulose(Li et al. 2015a) (PAC) are commonly used as rheological modifiers. These additives are helpful to obtain desired properties. However, few materials among these additives can simultaneously meet the demands of both technical and environmental factors. For example, these copolymer fluid loss additives might raise toxicity issues and could only be degraded at high temperatures(Aftab et al. 2020). The temperature resistance of Xanthan gum and PAC is limited with a temperature of lower than $120^{\circ} \mathrm{C}$ (Zhu et al. 2021). Thus, in order to minimize the environmental hazard and health risk as well as to meet the demand of drilling engineering, developing high-performance additives with environmentally friendly, biodegradable, and renewable properties is still a continuous effort and research focus, and even that one materials can exhibit multiple functions.

Cellulose has been always favored by researches due to their wide range of sources, low cost, facile modification, and suitable performance. They have a nanofibrillar structure and can form self-assembled systems in the nano and macro scale. In recent years, various cellulose nanoparticles, primarily including microfibrillated cellulose (MFC)(Kumar, Gupta and Gaikwad 2021), cellulose microcrystalline(MCC)(Ventura-Cruz and Tecante 2021), and cellulose nanocrystals (CNCs)(Ng et al. 2021), have been isolated from many cellulosic resources, such as wood, plants, marine animals, algae, and bacteria using different preparation methods. MFC can be produced by mechanical heating method(M Pääkkö 2007, Tsuguyuki Saito 2006). MFC has dimensions of $10 \sim 50 \mathrm{~nm}$ in width and several micrometers in length. CNCs, belonging to rod nanoparticles, can be fabricated using a strong acid hydrolysis method. Because of their nanoscale dimensions, high surface area, large aspect ratio, superior water retention 
ability, and self-assembling ability, these nanoparticles can form stable and viscous gel at a relatively low concentration, performing good dispersion stability, rheological property, and formability. Thus, these nanoparticles have been commonly applied in food, coatings, cosmetics, pharmaceuticals and materials industries. These intrinsically appealing features also enable cellulose nanoparticles to act as potential effective additives in oil industry. MFC and CNCs could perform rheological regulation and fluid loss control properties at room temperatures ( $\mathrm{Li}$ et al. 2015b, Villada et al. 2020). High quality cement materials could be prepared by adding MCC or CNCs(Montes et al. 2020, Kamasamudram, Ashraf and Landis 2021). Some modified nanocelluloses have been also expected to be an alternative for enhancing oil recovery.

In this study, carboxylated cellulose nanocrystals (CNCs) were developed as novel, renewable, biodegradable, effective, and environmentally friendly additives for BT-WBDFs application. CNCs showed multiple functions on improving the rheological, filtration, and inhibitive properties of BT-WBDFs at room and high temperatures. The interaction behaviors between CNCs and BT have been also studied in detail through measuring the colloidal stability of the fluids, observing the microstructure of BT/CNC suspensions and filter cakes, determining the wettability and water activity. Finally, the underlying mechanism on the performance improvement in BT-WBDFs was summarized.

\section{Materials And Methods}

2.1 Materials. CNCs (carboxylated, purity $\geq 99 \%$ ) were prepared and purchased from Guilin Qihong Technology Co., Ltd. BT (sodium form) was obtained by Energy Chemical (China). Sodium hydroxide was provided by Modern Oriental (Beijing) Technology Development (China) as a pH value adjuster. The shale cuttings were acquired from the oil field.

\subsection{Preparation of $\mathrm{BT} / \mathrm{CNC}$ suspensions}

BT $(6 \mathrm{~g})$ was added in deionized water $(300 \mathrm{ml})$ and vigorously stirred for $24 \mathrm{~h}$ to prepare BT suspensions. The BT concentration was always fixed at $2.0 \mathrm{wt} \%$ for all BT suspensions. Then, CNCs were added into BT suspensions for testing, and its concentration was varied at $0,0.25,0.5$, and $1.0 \mathrm{wt} \%$. In order to evaluate the influence of high temperatures on the property variations of $\mathrm{BT}$ suspensions after adding CNCs, an aging test was conducted by hot-rolling in a roller oven at a set temperature for $16 \mathrm{~h}$.

\subsection{Rheology analysis}

The rheological properties of BT/CNC suspensions were performed by a Haake Mars rheometer (Thermo Electron Corporation, Germany) with a cone-plate model. Before each measurement, the suspensions were vigorously stirred for 20 min. Approximately $1.5 \mathrm{ml}$ of the samples were carefully placed on the plate. The gap between the cone rotor and the plate is $53 \mu \mathrm{m}$, and the diameter of the cone rotor is $35 \mathrm{~mm}$, containing a cone angle of $1.0^{\circ}$. All tests began with a preshearing procedure $(2 \mathrm{~min}, \mathrm{Y}=$ $2000.0 \mathrm{~s}^{-1}$ ). Then, the apparent viscosity as a function of shear rate from 1000.0 to $0.1 \mathrm{~s}^{-1}$ was measured at $25^{\circ} \mathrm{C}$.

The rheological model of drilling fluids has an important and direct effect on cutting carrying capability, borehole cleaning, wellbore stabilization, and subsurface safety(Liu et al. 2021). For non-Newtonian drilling fluids, Bingham, power-law, and Herschel- Bulkely models are commonly used to fit the relationship between shear stress and shear rate(Ashrafizade 2010). The Bingham model is given by equation (1).

$$
\tau=\tau_{0}+\mu_{P V} \times \gamma
$$

where $\tau$ is the shear stress, $\tau_{0}$ is the yield stress, $\mu_{P V}$ is the plastic viscosity, and $\gamma$ is the shear rate. In the Bingham plastic model, the relationship between the shear stress and shear rate is linear. This model is simple and is adequate for some simple dispersions with high solid content and uniform particles. Afterwords, a power-law model is developed, as expressed by eqation (2).

$\tau=K \times \gamma^{n}(2)$ 
where $K$ is the consistency coefficient, and $n$ is the flow behavior index. Many polymer drilling fluids conform to the power-law model. The Herschel-Bulkely model is a power-law model with a yield point, as given by equation (3).

$\tau=\tau_{0}+K \gamma^{n}(3)$

Based on the shear stress and shear rate obtained from the rotating viscometer, the rheological curves of the set WBDF were fitted through a data processing system in Origin software.

\subsection{Filtration measurement}

Fluid loss for freshly prepared BT-WBDFs and those aged for $16 \mathrm{~h}$ were measured according to the American Petroleum Institute (API) guidelines for drilling fluids. About $240 \mathrm{ml}$ of the fluids were poured into a standard SD6 filter press (Qingdao Tongchun Petroleum Instrument Co., Ltd, China) equipped with quantitative filter paper. Afterwards, the fluids were pressed under a 0.69 MPa by using $\mathrm{N}_{2}$ gas chargers for 30 min and the fluid loss volumes were recorded.

\subsection{Inhibition measurement}

Linear swelling test. The inhibitive effect of CNCs on the swelling behavior of BT was evaluated by a CPZ-2dual-channel linear swelling meter (Tongchun, Qingdao). BT ( $5 \mathrm{~g}$ ) was pressed into a pellet under $10 \mathrm{MPa}$ pressure for $5 \mathrm{~min}$. Then, the BT pellet was placed on the instrument. $20 \mathrm{~mL}$ filtrate from filtration tests was added to immerse the BT pellet. Finally, the swelling height with time was recorded for $24 \mathrm{~h}$.

Hot-rolling recovery test. The inhibition performance of BT/CNC suspensions on the dispersion of shale cuttings at high temperatures was evaluated by hot-rolling recovery test. In this test, shale cuttings $(20 \mathrm{~g})$ between 6-10 mesh and $350 \mathrm{ml}$ BT/CNC suspensions were poured into a sealed jar together. Then, the jar was hot-rolled in a BGRL-5 roller furnace (Qingdao, China) at $140^{\circ} \mathrm{C}$ for $16 \mathrm{~h}$. After cooling to room temperature, the remaining shale cuttings were screened with a 40 -mesh sieve and washed repeatedly and gently with pure water. Finally, the recovered cuttings were dried at $75^{\circ} \mathrm{C}$ for $48 \mathrm{~h}$ and weighed. The shale recovery percentage was calculated by the following equation:

Shale recovery $=\left(\frac{m_{2}}{m_{1}}\right) \times 100 \%$

where $m_{1}$ was the mass of shale cuttings before hot-rolling and $m_{2}$ was the mass of shale cuttings remained after hot-rolling.

\subsection{Microstructural analysis of BT/CNC suspensions}

The morphology of the surface and cross section of the filter cakes from BT/CNC suspensions was observed using a SU8010 scanning electron microscopy (SEM) at an accelerating voltage of $5.0 \mathrm{kV}$. The structure of BT in CNC suspension was observed by a JEM-2100 transmission electron microscopy (TEM) at an accelerating voltage of $10 \mathrm{kV}$.

The water contact angles of filter cakes obtained form different BT/CNC suspensions were also measured. Deionized water was dropped onto the membrane with a micro-injector and the images were captured by a JC2000C contact angle tester.

The particle size distribution of BT/CNC suspensions was conducted using a Malvern Mastersizer 2000 particle size analyzer. The concentration of all samples was approximately $0.1 \mathrm{~g} \cdot \mathrm{L}^{-1}$. Each of the measurements was carried out at $25^{\circ} \mathrm{C}$.

The $\zeta$ potential of BT dispersion was measured by Malvern Zetasizer Nano series. The concentration of all samples in this test was approximately $2.0 \mathrm{~g} \cdot \mathrm{L}^{-1}$. Each of the measurements was repeated two times and the average value is used for accuracy.

The water activity $\left(\mathrm{a}_{\mathrm{w}}\right)$ of various inhibitor solutions was measured by HygroLab C1 at $25^{\circ} \mathrm{C}$. In this test, a $30 \mathrm{ml}$ sample was added into a disposable sample cup. Each measurement was repeated two times and the average was recorded.

\section{Results And Discussion}


The detailed characteristics of CNCs used in this study are summarized in Table 1, and their TEM micrographs are displayed in Figure 1. CNCs exhibited a short rod-like morphology with a width range of $5 \sim 20 \mathrm{~nm}$ and length of $50 \sim 100 \mathrm{~nm}$. Its aspect ratio was approximately between 2.5 20. After the carboxylation treatment, negatively charged carboxy groups were introduced on the surface of CNCs, which contributed to the colloidal stability under the electrostatic repulsion. The $\zeta$ potential also proved this result. CNCs performed $\zeta$ potential values of $-22.1 \mathrm{mV}$ at $\mathrm{pH} 6.95$ and $-31.5 \mathrm{mV}$ at $\mathrm{pH} 9.16$. Figure $1 \mathrm{~b}$ showed the apparent viscosity versus shear rate for CNC suspensions at concentrations of 0.5 and $1.0 \mathrm{wt} \%$. All CNC suspensions exhibited low viscosity at high shear rates, but high viscosity at low shear rates, which was commonly called as "shear-thinning" behavior(Yang Gang 2021). Moreover, the shear-thinning phenomenon became more significant as the concentration of CNCs increased from $0.5-1.0 \%$, which was desired performance of a drilling fluid. It can be inferred that CNCs can play a positive role on the rheological and filtration performance of BT-WBDFs.

Table 1

Physicochemical Characteristics of CNCs

\begin{tabular}{|lllllll|}
\hline sample & width $(\mathrm{nm})$ & length $(\mathrm{nm})$ & aspect ratio & 弓 potential $(\mathrm{mV})$ & $\mathrm{pH}$ & surface functional groups \\
\hline CNCs & $5 \sim 20$ & $50 \sim 100$ & $2.5 \sim 20$ & -22.1 & 6.95 & $-\mathrm{OH} /-\mathrm{COO}{ }^{-}$ \\
\hline
\end{tabular}

BT-WBDFs are greatly expected to be typical shear-thinning non-Newtonian fluids, which have high viscosity at low shear rates to suspend or carry cuttings from downhole, but low viscosity at high shear rates to reduce friction and assist rock breaking(Fagundes et al. 2018). CNCs were incorporated with BT suspensions to explore how profitable CNCs modified the rheological properties of BT-WBDFs. The concentrations of CNCs were varied in the range of 0.25 to $1.0 \mathrm{wt} \%$, while that of BT was fixed at $2.0 \mathrm{wt} \%$. Figure 2a,b showed the plots of viscosity versus shear rate for BT/CNC suspensions at different CNC concentrations and temperatures. All samples revealed a shear-thinning behavior in the whole range of shear rates. But BT and BT/CNC suspensions presented distinctive shear-thinning behaviors after high temperature treatment. At room temperature, BT suspension exhibited a predictable shear-thinning property, thereby being commonly used in WBDFs(Farag et al. 2019, Zou et al. 2019). The addition of CNCs effectively increased the viscosity of BT suspension and produced more remarkable shear-thinning behavior, which was conducive to optimizing drilling fluid performance. For example, at the shear rate of $1.0 \mathrm{~s}^{-1}$, the addition of $0.25,0.5$, and $1.0 \mathrm{wt} \%$ CNCs into BT suspension increased the apparent viscosity values from 201.59554 to 740.77179 , 1602.26233 , and $4702.94775 \mathrm{mPa} \cdot \mathrm{s}$, respectively. In comparison with the result at room temperature, the high temperature processing at $140^{\circ} \mathrm{C}$ sharply decreased the viscosity of pure BT suspension, but the viscosity of BT/CNC suspensions maintained highly values, and the shear-thinning behavior was more outstanding. Moreover, it can been seen that the shearthinning behavior for BT/CNC suspensions at room temperature was progressive, whereas that for BT/CNC suspensions at $140^{\circ} \mathrm{C}$ was nonprogressive, in which all BT/CNC suspensions displayed similar viscosity values at low shear rates, and progressive viscosity variation at high shear rates. For example, at a shear rate of $1.0 \mathrm{~s}^{-1}, \mathrm{BT} / \mathrm{CNC}$ suspensions with $0.25,0.5$, and $1.0 \mathrm{wt} \%$ of CNCs had viscosity values of $2353.05176,2851.86499$, and $3578.5708 \mathrm{mPa} \cdot \mathrm{s}$, respectively, while at a shear rate of $119 \mathrm{~s}^{-1}$, that were $21.98516,48.28335$, and $65.98486 \mathrm{mPa} \cdot \mathrm{s}$, respectively. Based on these observations, it can be concluded that CNCs can efficiently improve the shear- thinning properties of BT suspensions, even under high-temperature conditions.

Furtherly, the curves of shear stress versus shear rate for BT/CNC suspensions at different CNC concentrations were shown in Figure 2c,d. Similar to the viscosity results, the shear stress also enhanced with the increase in the concentration of CNCs. The Bingham plastic, power-law and Herschel-Bulkey models were applied to fit their shear stress-shear rate curves, and the corresponding fit parameters were listed in Table 2 . Whether room temperature or $140^{\circ} \mathrm{C}$, the Herschel-Bulkey model was more befitting for all shear stress-shear rate curves, compared with the Bingham plastic and power-law models, which was evidenced by the higher values of $\mathrm{R}^{2}$. In detail, after the addition of CNCs, the yield point values increased and the the flow behavior $n$ index decreased with the increase of CNC concentration. The yield point indicated the stress required to start the flow of drilling fluid(Syed M. Hussaini 1983), which could reflect the strength of grid structure between clay particles and CNCs(Ismail et al. 2016). The rheological modeling results further proved that CNCs had superior rheological modification ability, beneficial for transporting the cuttings and cleaning the wellbore(Liu et al. 2017). 
Table 2

Calculated Parameters for BT/CNC suspensions at different CNC concentrations using Bingham, Power-Law, and Herschel-Bulkley Models

\begin{tabular}{|c|c|c|c|c|c|c|c|c|c|}
\hline \multirow[t]{2}{*}{ models } & \multirow[t]{2}{*}{ parameters } & \multicolumn{4}{|c|}{$\mathrm{CNC}$ concentrations (wt \%) $/ 25^{\circ} \mathrm{C}$} & \multicolumn{4}{|c|}{$\mathrm{CNC}$ concentrations $(\mathrm{wt} \%) / 140^{\circ} \mathrm{C}$} \\
\hline & & 0 & 0.25 & 0.5 & 1.0 & 0 & 0.25 & 0.5 & 1.0 \\
\hline \multirow[t]{3}{*}{ Bingham } & $\tau_{0}$ & 0.20732 & 1.22334 & 2.4135 & 6.04269 & 0.13684 & 1.69228 & 3.22997 & 5.91782 \\
\hline & $\mu_{p}$ & 0.00281 & 0.00692 & 0.0118 & 0.0226 & 0.00455 & 0.00595 & 0.01065 & 0.01377 \\
\hline & $\mathrm{R}^{2}$ & 0.99703 & 0.97906 & 0.98173 & 0.98159 & 0.99744 & 0.97721 & 0.90405 & 0.99178 \\
\hline \multirow[t]{3}{*}{ Power-Law } & K & 0.01714 & 0.21803 & 0.48434 & 1.54894 & 0.0118 & 0.46404 & 0.9233 & 2.30946 \\
\hline & $n$ & 0.73984 & 0.50595 & 0.46988 & 0.40049 & 0.86143 & 0.38293 & 0.37155 & 0.28415 \\
\hline & $\mathrm{R}^{2}$ & 0.9895 & 0.98066 & 0.97835 & 0.96581 & 0.99891 & 0.95897 & 0.97517 & 0.90854 \\
\hline \multirow[t]{4}{*}{ Herschel-Bulkley } & $\tau_{y}$ & 0.16301 & 0.78231 & 1.65015 & 4.55636 & 0.05762 & 1.30569 & 1.10036 & 5.40477 \\
\hline & $K$ & 0.00525 & 0.04815 & 0.0836 & 0.16378 & 0.00901 & 0.04256 & 0.45219 & 0.05044 \\
\hline & $n$ & 0.90802 & 0.71707 & 0.71458 & 0.71131 & 0.8996 & 0.71313 & 0.46514 & 0.80994 \\
\hline & $R^{2}$ & 0.99862 & 0.9954 & 0.99928 & 0.99957 & 0.99942 & 0.99329 & 0.97909 & 0.99921 \\
\hline
\end{tabular}

Based on above measurement results, it can be speculated that there must be particular surface interactions between CNCs and BT layers. To verify the interactions and driving force between CNCs and BT, the macroscopic phenomenon and microstructures of BT/CNC suspensions were examined, respectively. As displayed in Figure 3, pure BT suspension could easily flow after being placed for 10h, while BT/CNC suspension added with $1.0 \mathrm{wt} \% \mathrm{CNCs}$ formed a whole piece of gel, indicating that BT/CNC had higher viscosity and stress. Moreover, after shaking for 10s, the formed BT/CNC gel could flow again. These distinctive phenomena between pure BT and BT/CNC suspensions demonstrated that CNCs had a strong gel formation capacity and a stiff network among BT layers, CNCs, and water molecules was created, which had a strong resistance to flow under shear force(Wang et al. 2018). The phase interactions between CNCs and BT layers were also observed by TEM (as shwon in Figure 4). BT hydrated in water and presented typical sheet-like structures (Figure 4a). In BT/CNC suspensions, CNCs were homogeneously dispersed in BT layers and they were entangled with each other (Figure 4b,c,d). The observations meant that CNCs were adsorbed onto the surface of BT particles and a stable colloidal structure was formed.

It was clear that BT was composed of a large number of plate-like crystal layers with permanent negative charges due to isomorphic substitutions on the faces and $\mathrm{pH}$-dependent charges developed on the surface hydroxyls at the edges(Anderson 2010). Due to the broken bonds of the octahedral $\mathrm{Al}-\mathrm{OH}$ and tetrahedral $\mathrm{Si}-\mathrm{OH}$ groups on the edges, some amphoteric sites are also existing and variable (either positive or negative) charges can develop at the edges depending on the pH. Besides, a positive charged edge could be also created due to the exposed octahedral Al layers when the pH value is less than 9 (Tombácz 2006, Avena 2003). In pure BT suspension, the ionic attraction between the negatively chared face and positively charged edge (edgeto-face attraction) could form a "house-of-cards" structure, which was responsible for the viscosity of BT suspensions(Li et al. 2020). Therefore, BT was commonly used as a ideal additive in drilling fluid. In BT/CNC suspension, CNCs would adsorb to the surface of BT layers via hydrogen bond between their hydroxyl groups, and ionic bond between the positively charged edges of BT layers and the negatively charged carboxy groups of CNCs. Meanwhile, in addition to the hydration and colloidal properties of BT itself, CNCs also had strong gel formation capacity, due to the high specific surface area and a large number of hydroxyl groups on the nanoparticle surface(Robert J. Crawford 2012). Then a large number of water molecules were bounded at the vicinity of $\mathrm{BT}$ and CNCs and a compact network was created, leading to a strong resistance to flow under shear force, even at high temperatures. Based on above interaction, a significnt improvement in the rheological properties was achieved. 
Filtration property is one of the most important properties for drilling fluids. The penetration of fluids into the formation always causes shale swelling and wellbore collapse. Furthermore, high temperatures may also destroy the hydration of clay and induce the flocculation of BT particles. Poor filter cakes with large thickness and high porosity formed on the wall of wellbore, resulting in large fluid loss volume and poor filtration property(Wang et al. 2021). As shown in Figure 5, the API fluid loss volumes of BT and $\mathrm{BT} / \mathrm{CNC}$ suspensions were measured at different temperatures. After treatment at high temperature of $140^{\circ} \mathrm{C}$, the fluid loss volume of pure $2.0 \mathrm{wt} \% \mathrm{BT}$ suspension increased from $41.0 \mathrm{ml}$ to $50.0 \mathrm{ml}$, revealing the detriment of high-temperature environments. By contrast, whether at room temperature or high temperatures, the addition of CNCs always improved the filtration performance of BT suspensions. The fluid loss volume of BT/CNC suspensions decreased as the concentration of CNCs increased, whereas the filter cakes also became thinner. No matter how CNCs worked, the filter cake was the final barrier to prevent filtration. It could be inferred that more suitable structure was formed in the filter cakes after adding CNCs as fluid loss reducer.

For drilling fluids, it is always thought that the filtration property is mainly determined by fluid viscosity and filter cake quality(S.M. Elkatatny 2011). Generally, high viscosity improves the fluid with the ability to against fluid loss(Borges et al. 2021). As analyzed in Figure 2a,b, the viscosity increased with increaseing the concentration of CNCs. However, the viscosity of BT/CNC suspensions at low shear rates under $140^{\circ} \mathrm{C}$ processing varied mildly, which was mainly related to the API fluid loss volume under static filtration condition. Therefore, increasing viscosity might be not the predominant factor affecting the filtration control property of CNCs. Then, the microstructure of filter cakes was further detected by SEM observation (as shown in Figure 6). At room temperature, it could be seen that both of the BT and BT/CNC presented satisfactory filter cakes with smooth and hydrated surface. Furthermore, the filter cakes obtained from BT/CNC suspensions exhibited more pronounced "colloid texture", like a polymer film. Similar phenomena also went for high tenperature environment of $140^{\circ} \mathrm{C}$. In order to observe the deposit structure of BT layers more clearly, the cross sections of these filter cakes were also investigated, as displayed in Figure 7. Obviously, the sections from BT/CNC were more regular and compact than that from pure BT, especially at high temperature. BT layers in BT/CNC deposited regularly under differential pressure and formed a clear lamellar structure, which was beneficial for producing thin and compact filter cakes.

Interestingly, we also found that the addition of CNCs improved the hydrophobicity of the filter cakes. As exhibited in Figure 8 , filter cakes from pure BT displayed strong hydrophilcity and had a water contact angle of approximate $27^{\circ}$ at room temperature and $140^{\circ} \mathrm{C}$. Adding CNCs dramatically enhanced the water contact angle of the filter cakes. For example, at a CNC concentration of $1.0 \mathrm{wt} \%$, the water contact angle of the filter cake obtained from BT/CNC suspensions was increased to 57.78 and $66.67^{\circ}$ at room temperature and $140^{\circ} \mathrm{C}$, respectively. The enhanced hydrophobic effect was favorable for preventing the invasion of water(Saparti et al. 2018). Besides, according to Wenzel's equation(Wang et al. 2015), the decrease of roughness can increase the contact angle measured on hydrophilic surface. The contact angle measurement also proved that the the addition of CNCs caused a more smooth surface on the filter cakes.

In short, based on the gel network among BT layers, CNCs, and immobilized water molecules, and the adsorption of CNCs on BT surface, BT deposited on the filter paper in a regular and compact layered structure, forming a more hydrophobic and smooth CNC polymer film on the surface. Finally, a high-quality filter cake was built and the fluid loss volume was sharply decreased.

In comparison with OBDFs, one of the deficiencies of WBDFs is the insufficient inhibition. There are more water in WBDFs and the fluids easily penetrate into the formation, resulting in clay hydration, swelling, and dispersion. Therefore, efficient shale inhibitors must be added in WBDFs (Muhammed, Olayiwola and Elkatatny 2021). The inhibition of CNCs was evaluated by linear swelling measurement and shale recovery test. As shown in Figure 9a, the swelling height curves of all samples exhibited a similar tendency with a dramatic increase rate within the initial period and a gradual slower growth as time proceeds, which was the typical swelling behavior of BT in aqueous solution. However, there was a gradual decrease in swelling height after CNCs were added as inhibitors. In detail, after immersing in water for $24 \mathrm{~h}$, the swelling height of BT pellet in pure water reached as high as $6.16 \mathrm{~mm}$. In comparison, the linear swelling heights of the BT pellets in filtrate obtained from BT/CNC suspensions at a CNC concentration of $0.25,0.5$, and $2.0 \mathrm{wt} \% \mathrm{CNCs}$ were $5.77,5.23$, and $4.00 \mathrm{~mm}$, respectively, displaying the inhibition property on BT swelling. Shale recovery test, as a standard method for evaluating the hydration dispersion of shale cuttings after hotrolling at a set temperature, was also conducted. As illustrated in Figure $9 \mathrm{~b}$, after aging at $140^{\circ} \mathrm{C}$, the shale recovery value of pure 
BT suspension was the lowest (29.4\%), which indicated the strong water sensitivity and dispersion of shales. Under the same conditions, the shale recovery values for BT/CNC suspensions with $0.25,0.5$, and $1.0 \mathrm{wt} \%$ of CNCs were $35.8,48.6$, and $55.25 \%$, respectively, suggesting the capability of CNCs for inhibiting the dispersion of the shales. Moreover, the results from hot-rolling shale recovery tests also demonstrated the temperature resistance of CNCs. The findings from linear swelling tests and shale recovery experiments were consistent. CNCs could used as valid inhibitors in WBDFs.

Generally, shale inhibitors achieved efficient inhibition through decreasing the interlayer spacing, such as $\mathrm{KCl}$, the oligomeric and polymeric amines with cationic groups(Anderson 2010), or encapsulation effect, such as various acrylamide copolymers(Zhang et al. 2018). Visibly, CNCs, as negatively charged solid-phase nanoparticles, might not inhibit BT by these approaches, presumably due to the interactions (gel network and adsorption) between CNCs and BT particles analyzed above, or whether there were other mechanisms. With these questions, the colloidal stability of BT was examined by measuring the $\zeta$ potential and particle size distribution. As shown in Figure 10, the addition of CNCs decreased the $\zeta$ potential efficiently. When the concentration of CNCs was $1.0 \mathrm{wt} \%$, the $\zeta$ potential of BT decreased from $-23.05 \mathrm{mV}$ to $-32.4 \mathrm{mV}$ at room temperature. The variation tendency of the $\zeta$ potential at $140^{\circ} \mathrm{C}$ was similar with that at room temperature. However, the whole values of the $\zeta$ potential at $140^{\circ} \mathrm{C}$ were higher, echoing the increase of fluid loss volume from 25 to $140^{\circ} \mathrm{C}$ in Figure 5 . Anyway, the cooperation of CNCs could increase the negative charges of BT particles and improve their stability.

Meanwhile, the particle size distribution of BT/CNC was also determined. As exhibited in Figure 11, the addition of CNCs didn't increase the particle size of BT, even showing a dispersion effect at small size range. These results demonstrated that CNCs didn't inhibit the hydration and swelling of BT by encapsulating the BT particles.

The $\zeta$ potential and particle size measurements indicated that CNCs could promote the stability of BT suspensions, which was contrary to the general inhibition mechanisms mentioned above. How does CNCs exert competent inhibition? In order to figure this out, the water activity of these samples were further evaluated (as listed in Table 3). Water activity is an important index in estimating shale/drilling fluid interactions, which can evaluate the hydration state of shale and its potential to adsorb or lose water. Drilling fluids with lower water activity than shale can reduce the osmotic pressure between drilling fluids and the formation fluids, finally preventing water from invading shales(Chenevert 1970, Jianguo Zhang 2008). From the Table 3, the water activity of BT samples decreased gradually with increasing the concentration of CNCs from 0 to $1.0 \%$. In fact, these results should be foreseeable. The rheological tests concluded that BT, CNCs and immobilized water molecules formed stiff gel network. This network could efficiently bound the water molecules and reduce the free water, thereby decreasing the water activity. Moreover, the filtration measurements indicated that CNCs could contribute to creating a thin, compact, and low-hydrophilicity filter cake. The plugging and wettability modification abilities of CNCs aslo restrained the penetration of free water into the clay or shales. Under these comprehensive actions, CNCs presented the inhibition well.

Table 3

The water activity of BT/CNC suspensions at different CNC concentrations after aging at $25^{\circ} \mathrm{C}$ and $140^{\circ} \mathrm{C}$

\begin{tabular}{|lll|}
\hline sample & \multicolumn{2}{c|}{ water activity /aw } \\
\cline { 2 - 3 } & $\mathbf{2 5 ^ { \circ } \mathrm { C }}$ & $\mathbf{1 4 0 ^ { \circ } \mathrm { C }}$ \\
\hline $\mathrm{BT}$ & 0.968 & 0.964 \\
\hline $\mathrm{BT}+0.25 \% \mathrm{CNCs}$ & 0.953 & 0.954 \\
\hline $\mathrm{BT}+0.5 \% \mathrm{CNCs}$ & 0.933 & 0.932 \\
\hline $\mathrm{BT}+1.0 \% \mathrm{CNCs}$ & 0.927 & 0.931 \\
\hline
\end{tabular}

In summary, the modification effect of CNCs on the properties of BT-WBDFs and the interaction mechanism were concluded and listed in Table 4, which was also clearly described by the schematic diagrams, as shown in Figure 12. BT platelet was negatively charged on the flat surface and positively charged on the edge. These plates could be linked together via face-to-face (FF), edge- 
to-edge (EE), and edge-to-face (EF) attractions. The edge-to-face attraction formed a "house-of-cards" structure, which was responsible for the viscosity of BT suspensions. When the CNCs were employed in BT suspensions, one hand, CNCs adsorbed on the surface of BT through hydrogen bond and electrostatic interaction. The BT platelet and CNCs interwove with each other and formed stable "house of cards" structure at low shear rates and dispersed structure at high shear rates. On the other hand, due to the strong gel formation capacity of CNCs, a stiff network between BT layer, CNCs, and water molecules was created. Finally, BT/CNC suspensions exhibited excellent "shear- thinning" property. Based on the prominent rheological properties and plugging function of CNCs, BT/CNC deposited on the filter paper with a compact layered structure and constituted a "film" with improved hydrophobic ability, thereby resulting in a thin and compact filter cake and low filtration volume. Meanwhile, under the actions of CNCs, BT/CNC suspensions kept colloidal stability with high viscosity, displayed lower water activity, and prevented the penetration of free water, exerting competent inhibition on hydration and dispersion of clays. As a result, CNCs performed great potential to be used as efficient and environmental-friendly rheological modifier, fluid loss agent, and clay inhibitor for BT-WBDFs.

Table 4

The functions and mechanisms of CNCs on improving the peoperties of BT-WBDFs

\begin{tabular}{|c|c|c|}
\hline & Effect & Mechanism \\
\hline \multirow[t]{2}{*}{$\begin{array}{l}\text { Rheological } \\
\text { property }\end{array}$} & $\begin{array}{l}\text { The addition of } 1.0 \text { wt \% CNCs increased the yield point of BT suspension from } \\
0.13684 \text { to } 5.91782 \mathrm{~Pa} \text { at } 140^{\circ} \mathrm{C} \text {. }\end{array}$ & $\begin{array}{l}\text { Adsorption of CNCs } \\
\text { on BT; }\end{array}$ \\
\hline & & $\begin{array}{l}\text { Stiff network } \\
\text { between BT layer, } \\
\text { CNCs, and water } \\
\text { molecules. }\end{array}$ \\
\hline \multirow[t]{3}{*}{$\begin{array}{l}\text { Filtration } \\
\text { property }\end{array}$} & $\begin{array}{l}\text { The addition of } 1.0 \text { wt } \% \text { CNCs decreased the API fluid loss volume of BT suspension } \\
\text { from } 50.0 \text { to } 16.0 \mathrm{ml} \text { at } 140^{\circ} \mathrm{C} \text {. }\end{array}$ & $\begin{array}{l}\text { Thin and compact } \\
\text { filter cakes with } \\
\text { layered structure; }\end{array}$ \\
\hline & & $\begin{array}{l}\text { Improve the } \\
\text { hydrophobic } \\
\text { property of filter } \\
\text { cakes; }\end{array}$ \\
\hline & & $\begin{array}{l}\text { Plugging effect of } \\
\text { CNCS. }\end{array}$ \\
\hline \multirow[t]{4}{*}{$\begin{array}{l}\text { Inhibition } \\
\text { property }\end{array}$} & $\begin{array}{l}\text { The addition of } 1.0 \mathrm{wt} \% \mathrm{CNCs} \text { decreased the swelling height of BT in filtrate from } \\
6.16 \text { to } 4.00 \mathrm{~mm} \text { at room temperature. The addition of } 1.0 \mathrm{wt} \% \mathrm{CNCs} \text { increased the } \\
\text { shale recovery of BT suspension from } 29.40 \text { to } 55.25 \% \text { at } 140^{\circ} \mathrm{C} \text {. }\end{array}$ & $\begin{array}{l}\text { Adsorption of CNCs } \\
\text { on BT; }\end{array}$ \\
\hline & & $\begin{array}{l}\text { Stiff network } \\
\text { between BT layer, } \\
\text { CNCs, and water } \\
\text { molecules; }\end{array}$ \\
\hline & & Low water activity; \\
\hline & & Plugging effect. \\
\hline
\end{tabular}

\section{Conclusion}

Carboxylated cellulose nanocrystals (CNCs) were selected as efficient and environmentally friendly additives to improve the rheological, filtration, and inhibitive properties of BT-WBDFs. CNCs were modified by carboxylation reaction, displaying small size, negative surface charge, good colloidal stability, and prominent shear-thinning behavior. The experimental results indicated that BT/CNC suspensions had superior rheological properties, low fluid loss volumes, and effective inhibition, even at $140^{\circ} \mathrm{C}$. CNCs could attach to the surface of BT via hydrogen bond and ionic bond. A stiff gel network between CNCs, BT, and vicinal water molecules was observed and proved, which had a strong resistance to flow under shear force, leading to a significant improvement in the rheological properties, including the viscosity, shear force, and yield point. Moreover, under the differential pressure, the improved viscosity, the created gel network as well as the formation of CNC films remarkably reduced the fluid loss volume, forming a thin, compact, and less hydrophilic filter cake with layered structure. Finally, benefiting from the gel network and outstanding filtration property, BT/CNC performed low water activity and plugging effect, which could prevent the 
penetration of free water into the shales and borehole well, therefore exerting satisfactory inhibitive performance for shale formations. CNCs could be used as multi-functional, and environmentally friendly additives in BT-WBDFs. This research demonstrates the effectiveness of CNCs in enhancing the performances of BT-WBDFs, offering a pathway for designing a new generation of additives in drilling fluid applications.

\section{Declarations}

\section{Acknowledgments}

This collaborative study was carried out with support from the China Postdoctoral Science Foundation, and CNPC Chuanqing Drilling Engineering Company Limited, China.

\section{References}

1. Adeleye Sanmi Apaleke A, A.-M. M, Enamul Hossain (2012) Drilling Fluid: State of The Art and Future Trend. The North Africa Technical Conference and Exhibition, SPE 149555

2. Aftab A, Ali M, Sahito MF, Mohanty US, Jha NK, Akhondzadeh H, Azhar MR, Ismail AR, Keshavarz A, Iglauer S (2020) Environmental Friendliness and High Performance of Multifunctional Tween 80/ZnO-Nanoparticles-Added Water-Based Drilling Fluid: An Experimental Approach, 8. ACS Sustainable Chemistry \& Engineering, pp 11224-11243

3. Agwu OE, Akpabio JU, Ekpenyong ME, Inyang UG, Asuquo DE, Eyoh IJ, Adeoye OS (2021) A critical review of drilling mud rheological models. J Petrol Sci Eng 203:108659

4. Akpan EU, Enyi GC, Nasr G, Yahaya AA, Ahmadu AA, Saidu B (2019) Water-based drilling fluids for high-temperature applications and water-sensitive and dispersible shale formations. J Petrol Sci Eng 175:1028-1038

5. Amanullah M, Ramasamy J, Al-Arfaj MK, Aramco S (2016) Application of an indigenous eco-friendly raw material as fluid loss additive. J Petrol Sci Eng 139:191-197

6. Anderson RL, Ratcliffe I, Greenwell HC, Williams PA, Cliffe S, Coveney PV (2010) Clay swelling - A challenge in the oilfield. Earth Sci Rev 98:201-216

7. Ashrafizade MN, a. SN (2010) Novel Equation for the Prediction of Rheological Parameters of Drilling Fluids in an Annulus. Industrial Engineering Chemistry Research 49:3374-3385

8. Avena M (2003) Proton binding at clay surfaces in water. Appl Clay Sci 24:3-9

9. Borges RFO, Oechsler BF, Oliveira BR, Andrade LDR, Calçada LA, Scheid CM, Calado V (2021) Reparameterization of static filtration model of aqueous-based drilling fluids for simultaneous estimation of compressible mudcake parameters. Powder Technol 386:120-135

10. Chenevert M (1970) Shale Control with Balanced-Activity Oil-Continuous Muds. J Petrol Technol 22:1309-1316

11. Fagundes FM, Santos NBC, Damasceno JJR, Arouca FO (2018) Study on the stability of a shear-thinning suspension used in oil well drilling, 73. Oil \& Gas Sciences and Technology - Revue d'IFP Energies nouvelles, p 10

12. Farag RM, Salem AM, El-Midany AA, El-Mofty SE (2019) Justifying API Bentonite Rheological Behavior Through Its Forming Size Fractions, 37. Mining, Metallurgy \& Exploration, pp 537-542

13. Igwilo KC, Uwaezuoke N, Onyekwere RK, Amaefule VC, Durogbitan AA, Ikoro VG (2020) Comparative assessment of Mucuna solannie as an alternative fluid loss control material in synthetic drilling fluid design. Journal of Petroleum Exploration and Production Technology

14. Ismail AR, Wan Sulaiman WR, Jaafar MZ, Ismail I, E. Sabu Hera (2016) Nanoparticles Performance as Fluid Loss Additives in Water Based Drilling Fluids. Mater Sci Forum 864:189-193

15. Zhang J, David JCR, Clark E (2008) Stressed-Shale Drilling Strategy - Water-Activity Design Improves Drilling Performance. Society of Petroleum Engineers 23:385-393

16. Kamasamudram KS, Ashraf W, Landis EN (2021) Cellulose nanofibrils with and without nanosilica for the performance enhancement of Portland cement systems. Constr Build Mater 285:121547

Page $10 / 17$ 
17. Kumar A, Gupta V, Gaikwad KK (2021) Microfibrillated cellulose from pine cone: extraction, properties, and characterization. Biomass Conversion and Biorefinery

18. Li M-C, Wu Q, Song K, De Hoop CF, Lee S, Qing Y, Wu Y (2015a) Cellulose Nanocrystals and Polyanionic Cellulose as Additives in Bentonite Water-Based Drilling Fluids: Rheological Modeling and Filtration Mechanisms, 55. Industrial \& Engineering Chemistry Research, pp 133-143

19. Li MC, Wu Q, Song K, Qing Y, Wu Y (2015b) Cellulose nanoparticles as modifiers for rheology and fluid loss in bentonite water-based fluids. ACS Appl Mater Interfaces 7:5006-5016

20. Li X, Jiang G, Shen X, Li G (2020) Application of Tea Polyphenols as a Biodegradable Fluid Loss Additive and Study of the Filtration Mechanism. ACS Omega 5:3453-3461

21. Liu F, Jiang G-C, Wang K, Wang J (2017) Laponite nanoparticle as a multi-functional additive in water-based drilling fluids. Journal of Materials Science 52:12266-12278

22. Liu F, Jiang G, Peng S, He Y, Wang J (2016) Amphoteric Polymer as an Anti-calcium Contamination Fluid-Loss Additive in Water-Based Drilling Fluids. Energy Fuels 30:7221-7228

23. Liu L, Pu X, Tao H, Deng Q, Luo A (2018) Synthesis and characterization of comb-shaped copolymer as a filtration reducer and comparison with counterparts. RSC Advances 8:11424-11435

24. Liu N, Zhang D, Gao H, Hu Y, Duan L (2021) Real-Time Measurement of Drilling Fluid Rheological Properties: A Review. Sensors (Basel), 21

25. Attia M, Elsorafy W, D'Angelo S (2010) New Engineered Approach to Replace Oil-Based Drilling Fluids with High Performance Water-Based Drilling Fluids in Mediterranean Sea. North Africa Technical Conference \& Exhibition, SPE 127826

26. Pääkkö M, Kosonen MA,H, Nykänen A, Ahola S, Osterberg M, Ruokolainen J, Laine J, Larsson PT, Ikkala O, T Lindström (2007) Enzymatic Hydrolysis Combined with Mechanical Shearing and High-Pressure Homogenization for Nanoscale Cellulose Fibrils and Strong Gels, 8. Biomacromolecules, pp 1934-1941

27. Montes F, Fu T, Youngblood JP, Weiss J (2020) Rheological impact of using cellulose nanocrystals (CNC) in cement pastes. Constr Build Mater 235:117497

28. Muhammed NS, Olayiwola T, Elkatatny S (2021) A review on clay chemistry, characterization and shale inhibitors for waterbased drilling fluids. J Petrol Sci Eng 206:109043

29. Ng LY, Wong TJ, Ng CY, Amelia CKM (2021) A review on cellulose nanocrystals production and characterization methods from Elaeis guineensis empty fruit bunches. Arabian Journal of Chemistry 14:103339

30. Patel A, Stamatakis S, Young S, Friedheim J (2007) Advances in Inhibitive Water-Based Drilling Fluids-Can They Replace Oil-Based Muds? SPE International Symposium on Oilfield Chemistry, SPE 106476

31. Caenn R, Chillingar G (1996) Drilling fluids: State of the art. Journal of Petroleum Science Engineering 14:221-230

32. Robert J, Crawford KJE, Lindhoud S, Scott JL, Gianfranco Unali (2012) Formation of shear thinning gels from partially oxidised cellulose nanofibril. Green Chem, 14, 300-303

33. Elkatatny SM, M. A. M., and Nasr-El-Din HA (2011) A New technique to Characterize Drilling Fluid Filter Cake. SPE European Formation Damage Conference, SPE 144098

34. Saparti M, Jali N, Rohani R, Chung YT, Wan WR, Sulaiman (2018) Hydrophobic Nanosilica as Fluid Loss Control Additive for High Performance Water- Based Drilling Fluids, SI1. Jurnal Kejuruteraan, pp 75-85

35. Chegny SJ, a. KT N. A (2008) The Possibility of Replacing OBMs with Emulsified Glycol Mud Systems in Drilling LowPressure Zones of Iranian Oilfields. IADC/SPE Asia Pacific Drilling Technology Conference IADC/SPE 114067

36. Sun H, Xin Y, Hu Y (2013) Preparation Mechanism and Experimental Results on Deep Thermophilic and Halotolerant Solidsfree Drilling Fluids. Asian J Chem 25:2866-2868

37. Syed M, Hussaini JJA (1983) Experimental Study of Drilled Cuttings Transport Using Common Drilling Muds SPE Journal, $2311-20$

38. Tombácz E, Szekeres M (2006) Surface charge heterogeneity of kaolinite in aqueous suspension in comparison with montmorillonite. Appl Clay Sci 34:105-124

Page $11 / 17$ 
39. Tsuguyuki Saito YN, Putaux J-L, Vignon M, Isogai A (2006) Homogeneous Suspensions of Individualized Microfibrils from TEMPO-Catalyzed Oxidation of Native Cellulose. Biomacromol 7:1687-1691

40. Ventura-Cruz S, Tecante A (2021) Nanocellulose and microcrystalline cellulose from agricultural waste: Review on isolation and application as reinforcement in polymeric matrices. Food Hydrocolloids 118:106771

41. Villada Y, Gallardo F, Erdmann E, Casis N, Olivares L, Estenoz D (2017) Functional characterization on colloidal suspensions containing xanthan gum (XGD) and polyanionic cellulose (PAC) used in drilling fluids for a shale formation. Appl Clay Sci 149:59-66

42. Villada Y, Iglesias MC, Olivares ML, Casis N, Zhu J, Peresin MS, Estenoz D (2020) Di-carboxylic acid cellulose nanofibril (DCA-CNF) as an additive in water-based drilling fluids (WBMs) applied to shale formations. Cellulose 28:417-436

43. Wang G, Jiang G, Yang J, Yang L, Li X, He Y, Chang X (2021) Novel N,N-dimethylacrylamide copolymer containing multiple rigid comonomers as a filtrate reducer in water-based drilling fluids and mechanism study. J Appl Polym Sci 138:51001

44. Wang K, Jiang G, Liu F, Yang L, Ni X, Wang J (2018) Magnesium aluminum silicate nanoparticles as a high-performance rheological modifier in water-based drilling fluids. Appl Clay Sci 161:427-435

45. Wang X-S, Fan X-B, Cui S-W, Sun Z-W, Xu S-H, Zhu R-Z, Hu A, Zhou L (2015) A Generalized Wenzel's Equation for Wetting of Droplets on Rough Substrates with Air Bubbles Trapped at the Solid-Liquid Interface. J Comput Theor Nanosci 12:37873791

46. Yang Gang ZT, Qi-Hao C, Zhang Hui-Chen (2021) Molecular dynamics simulation on shear thinning characteristics of nonNewtonian fluids. Acta Physica Sinica 70:124701

47. Yang L, Jiang G, Shi Y, Lin X, Yang X (2017) Application of ionic liquid to a high-performance calcium-resistant additive for filtration control of bentonite/water-based drilling fluids. Journal of Materials Science 52:6362-6375

48. Yao R, Jiang G, Li W, Deng T, Zhang H (2014) Effect of water-based drilling fluid components on filter cake structure. Powder Technol 262:51-61

49. Zhang X, Jiang G, Dong T, Wang L, Li X, Wang G (2018) An amphoteric polymer as a shale borehole stabilizer in water-based drilling fluids. J Petrol Sci Eng 170:112-120

50. Zhu W, Zheng X, Shi J, Wang Y (2021) A high-temperature resistant colloid gas aphron drilling fluid system prepared by using a novel graft copolymer xanthan gum-AA/AM/AMPS. J Petrol Sci Eng 205:108821

51. Zou Z, Zhou F, Wang Q, Zhao Q, Tian Y, Liu W, Chen L (2019) Enhanced dispersive stability of bentonite suspension in saline water-based mud. Colloids Surf A 579:123589

\section{Figures}
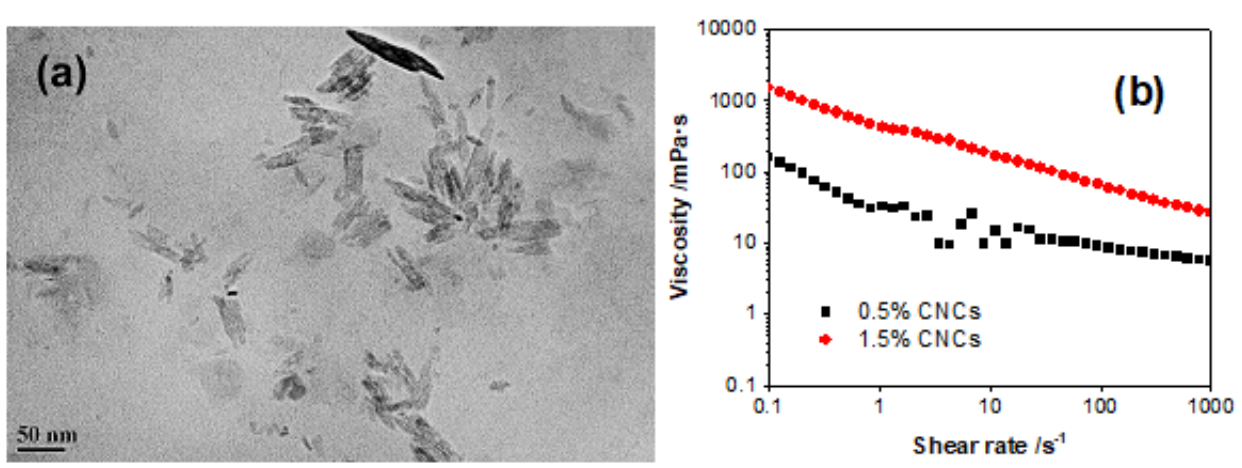

\section{Figure 1}

TEM micrographs of CNCs (a) and shear-thinning behaviors (b) of CNCs dispersions 

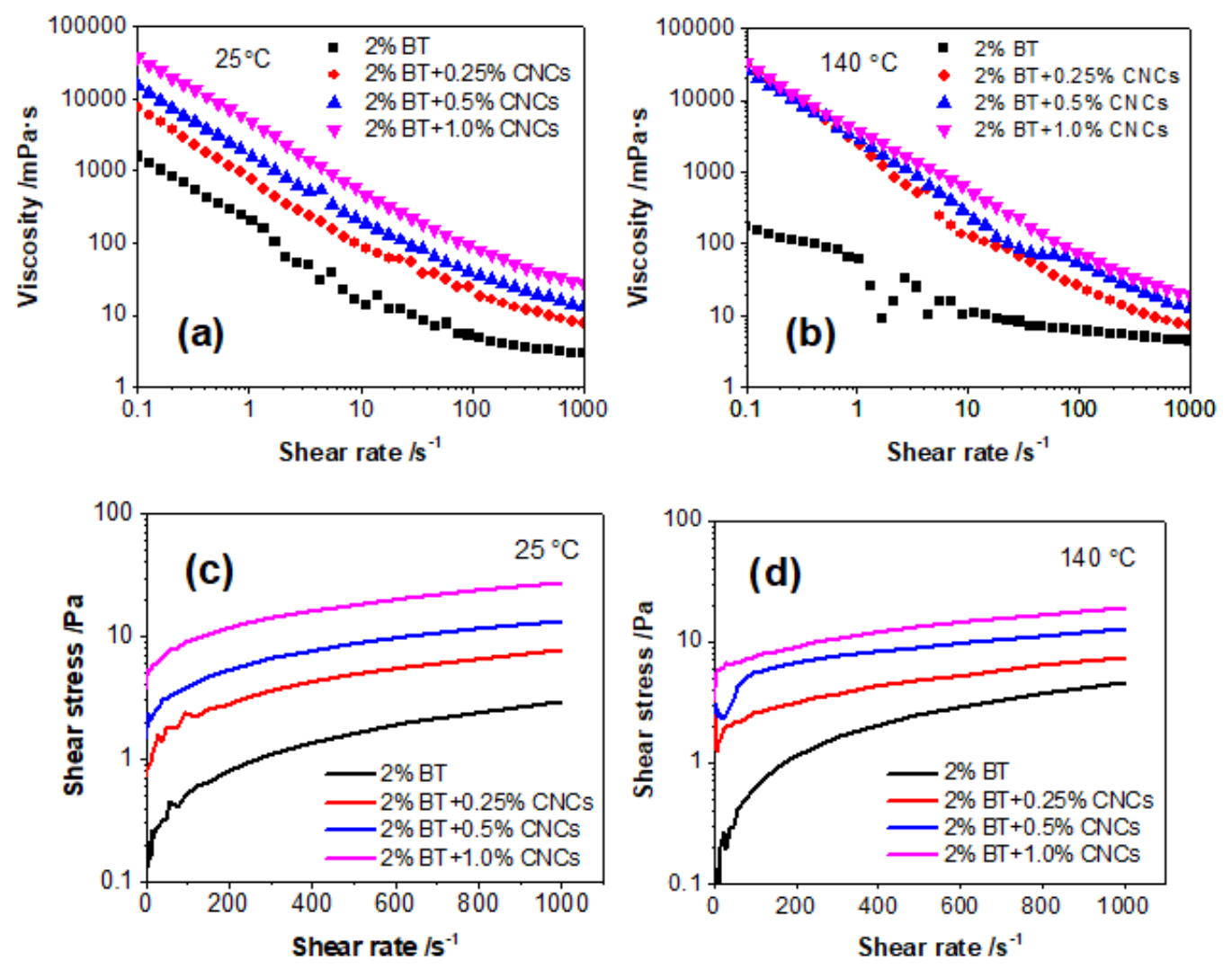

Figure 2

The plots of viscosity versus shear rate for BT/CNC suspensions with different CNC concentrations of $0,0.25 .0 .5$, and 1.0 wt $\%$ at $25^{\circ} \mathrm{C}$ (a) and $140^{\circ} \mathrm{C}$ (b). The curves of shear stress versus shear rate for BT/CNC suspensions with different CNC concentrations at $25^{\circ} \mathrm{C}(\mathrm{c})$ and $140^{\circ} \mathrm{C}(\mathrm{d})$.

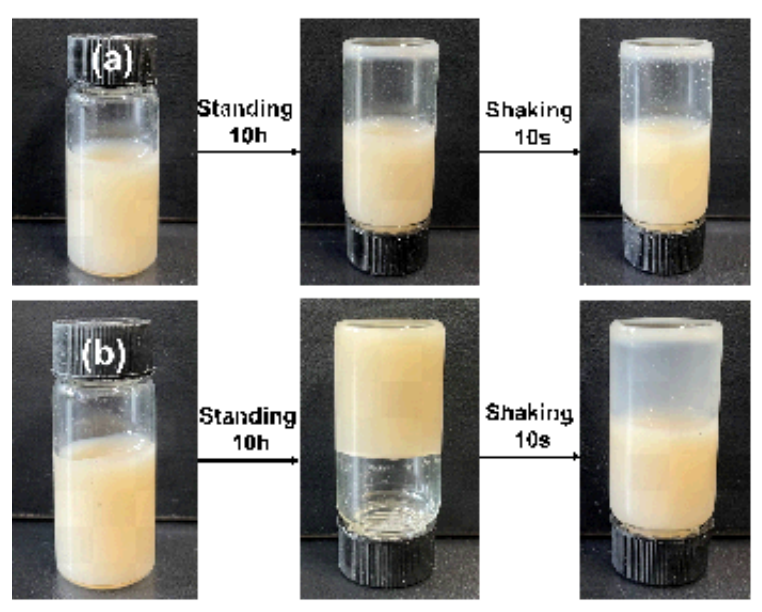

\section{Figure 3}

Gel structure of pure BT suspension (a), and BT/CNC suspension added 1.0 wt \% CNCs (b) 

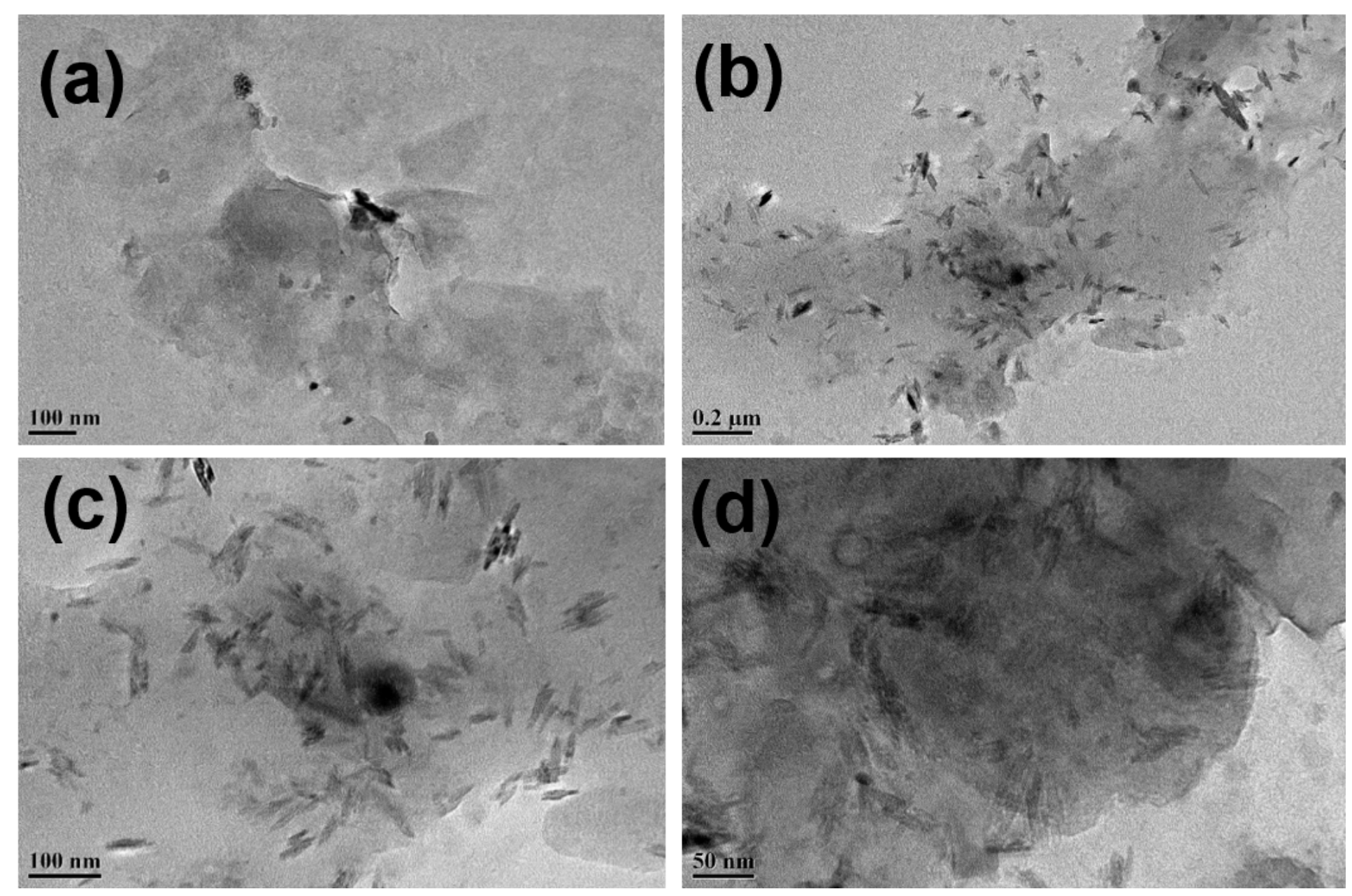

Figure 4

TEM micrographs of BT (a), and BT/CNC (b). The concentration of CNCs in BT suspension was $1.0 \mathrm{wt} \%$, and then the fluids were diluted 10 times using deionized water.
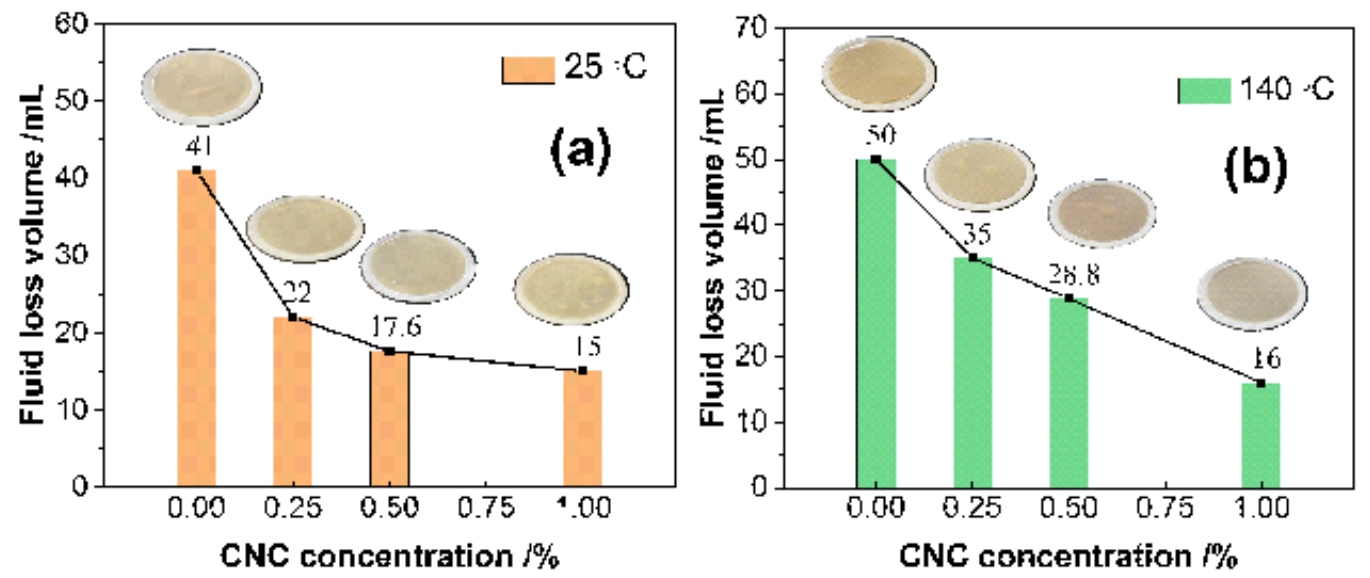

\section{Figure 5}

The fluid loss volumes and filter cakes of BT/CNC suspensions at CNCs concentrations of $0,0.25 .0 .5$, and 1.0 wt $\%$ after hot rolling at $25^{\circ} \mathrm{C}(\mathrm{a})$ and $140^{\circ} \mathrm{C}(\mathrm{b})$. 


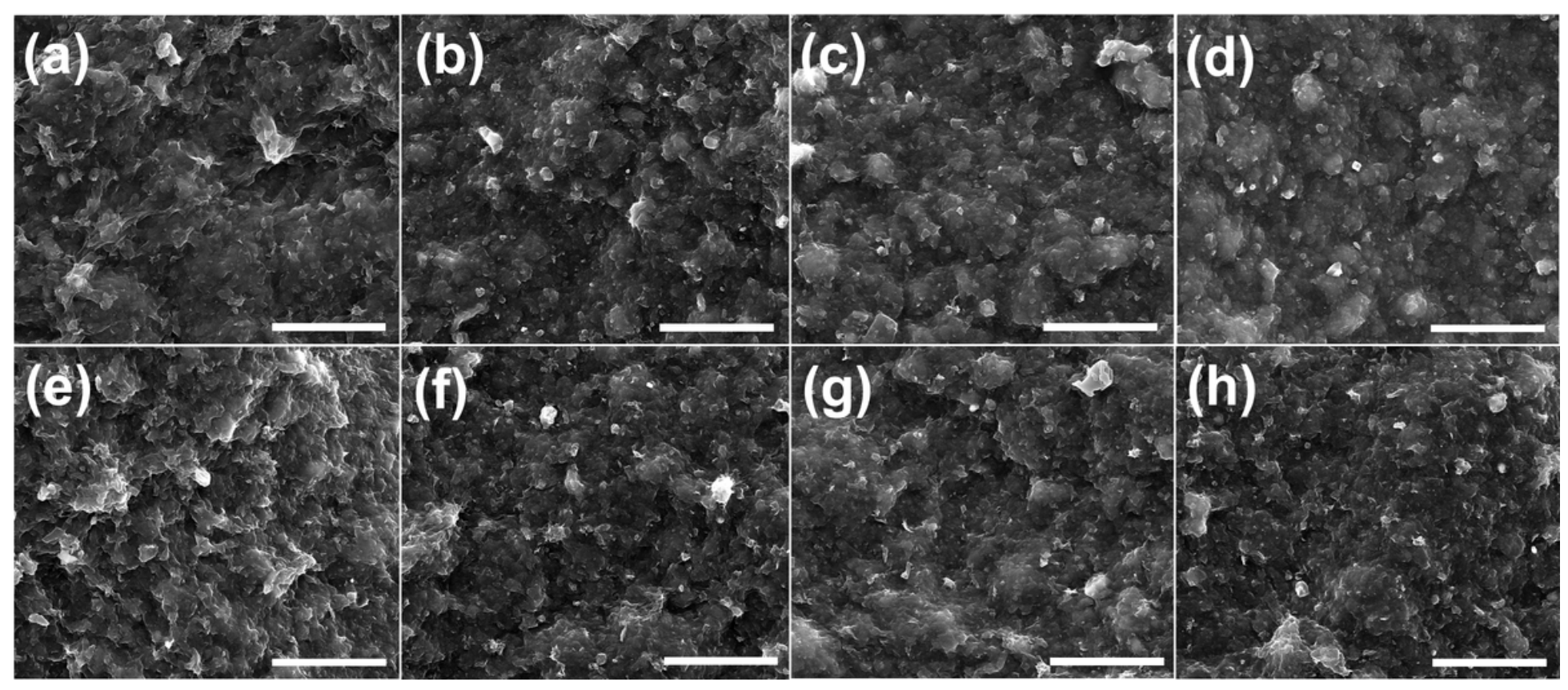

\section{Figure 6}

SEM micrographs of dried filter cakes obtained from BT/CNC suspensions at CNC concentrations of 0 (a), $0.25 \%$ (b), $0.5 \%$ (c), and $1.0 \% \mathrm{CNCs}$ at $25^{\circ} \mathrm{C}$. SEM micrographs (e-h) were obtained from BT/CNC after aging at $140{ }^{\circ} \mathrm{C}$ for $16 \mathrm{~h}$. The graph scale length was $30 \mu \mathrm{m}$.

2

\section{Figure 7}

Cross-sectional SEM micrographs of filter cakes from different BT/CNC suspensions: (a) BT at $25^{\circ} \mathrm{C}$, (b) BT+ 1.0 wt \% CNCs at $25^{\circ} \mathrm{C}$, (c) BT after aging at $140{ }^{\circ} \mathrm{C}$, (d) BT+ $1.0 \mathrm{wt} \% \mathrm{CNCs}$ after aging at $140^{\circ} \mathrm{C}$. The graph scale length was $5 \mu \mathrm{m}$.

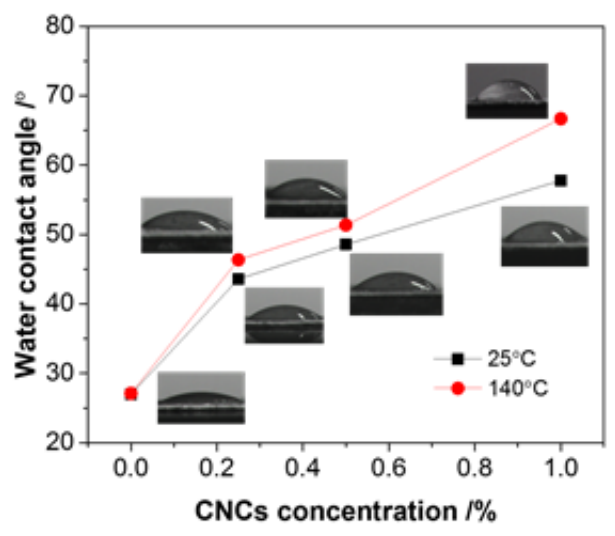

\section{Figure 8}

The water contact angels of the filter cakes obtained from BT/CNC suspensions at different CNC concentrations after aging at 25 and $140^{\circ} \mathrm{C}$. 

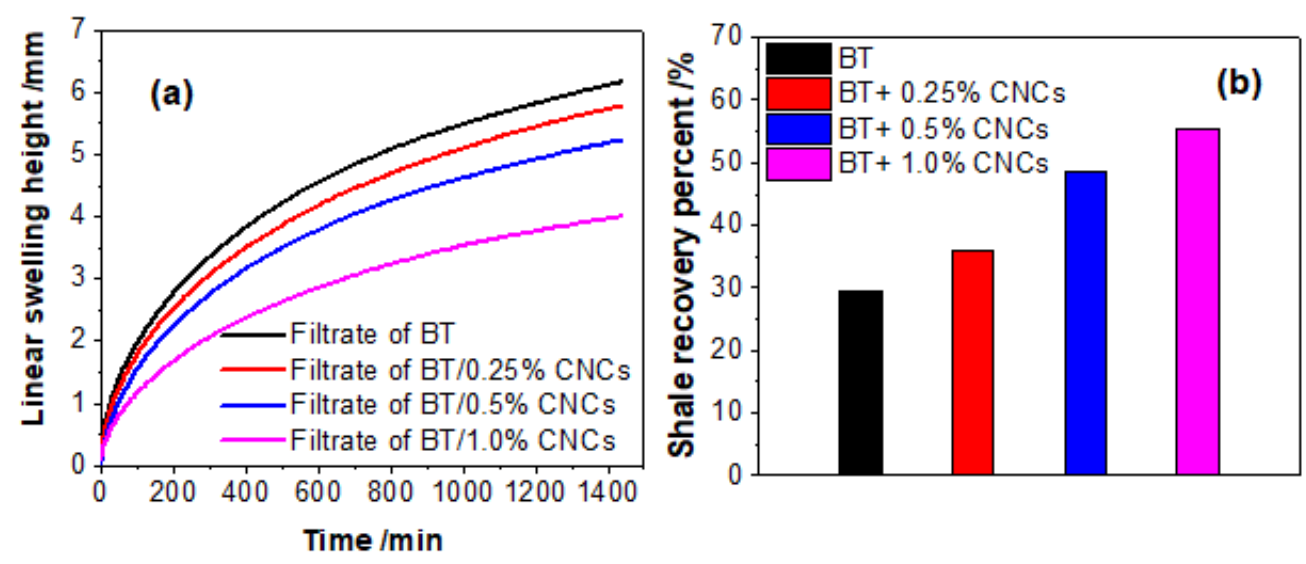

\section{Figure 9}

The linear swelling height of filtrate (a) from BT/CNC suspensions, and the shale recovery (b) of BT/CNC suspensions as a function of the CNC concentration.

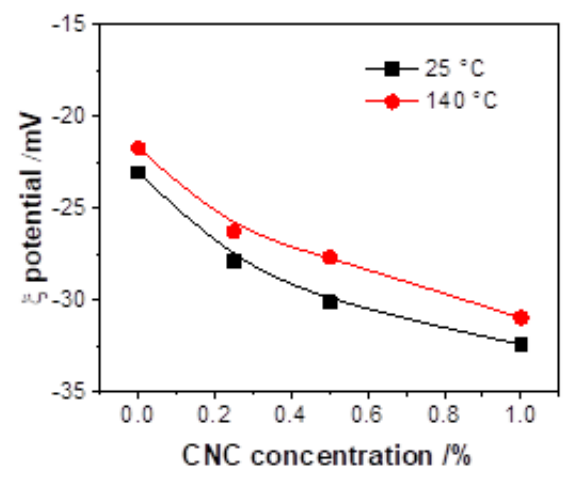

Figure 10

The $\zeta$ potential of BT/CNC suspensions at different CNC concentrations after treatment at $25^{\circ} \mathrm{C}$ and $140{ }^{\circ} \mathrm{C}$.
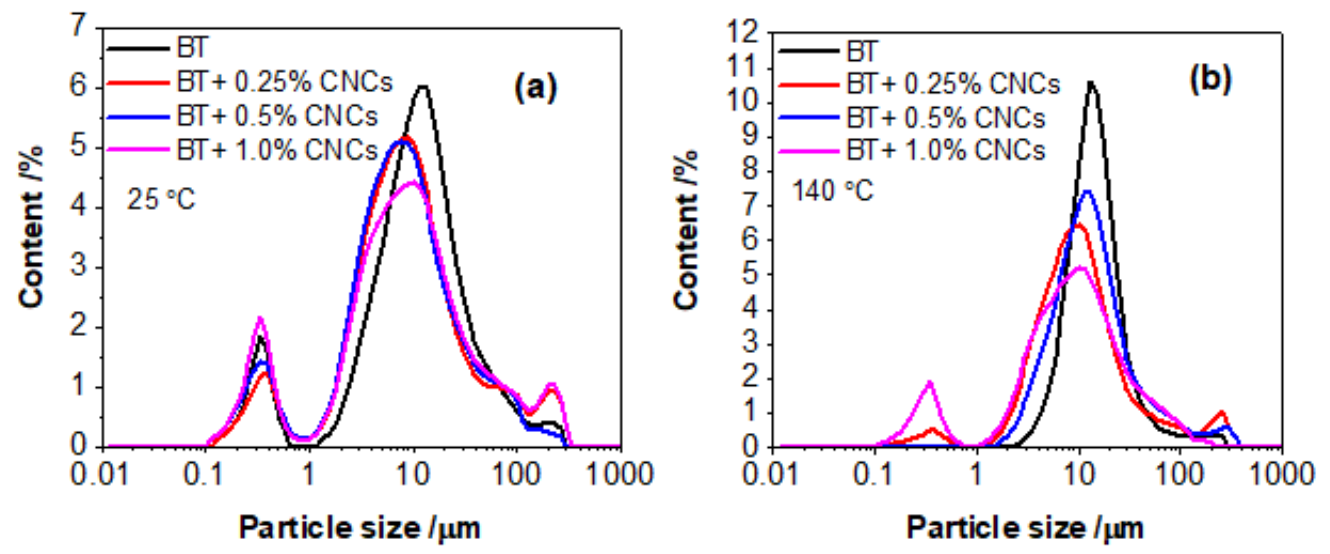

Figure 11

The particle size distribution of $\mathrm{BT} / \mathrm{CNC}$ suspensions at different $\mathrm{CNC}$ concentrations after treatment at $25^{\circ} \mathrm{C}(\mathrm{a})$ and $140{ }^{\circ} \mathrm{C}(\mathrm{b})$. 

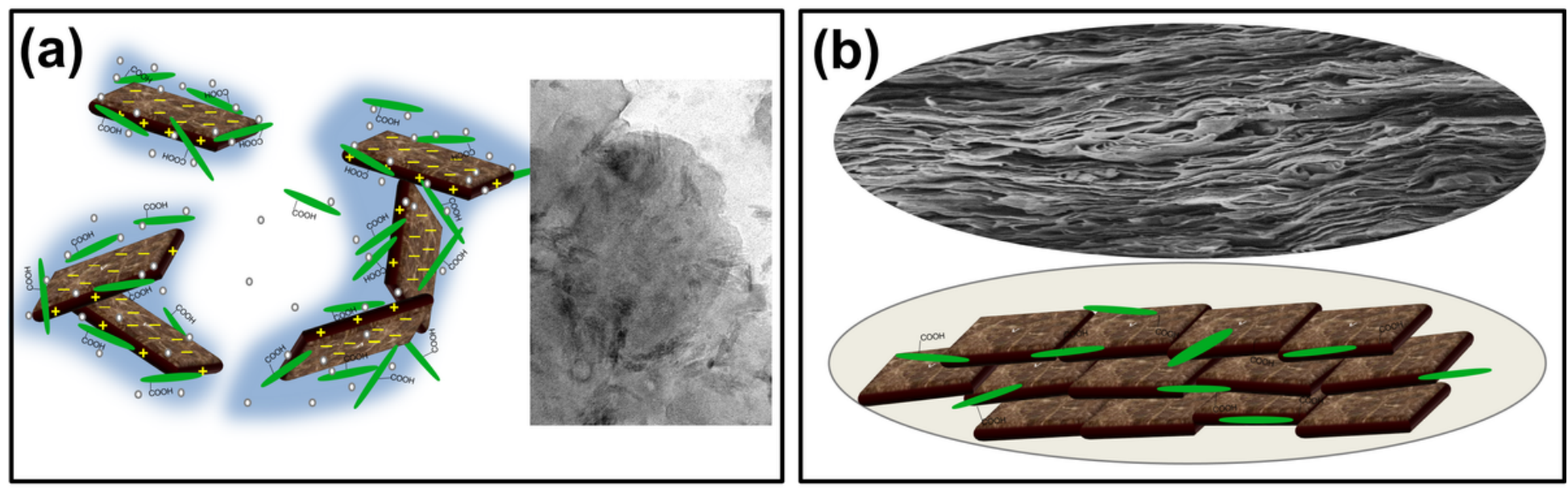

$\mathrm{COOH}$ CNCs

Figure 12

Schematic diagrams of the interaction between BT and CNCs. 